\title{
Controlling the work function of a diamond-like carbon surface by fluorination with $\mathrm{XeF}_{2}$
}

\author{
Ana Tarditi and Petro Kondratyuk \\ Department of Chemical Engineering, Carnegie Mellon University, 5000 Forbes Ave., Pittsburgh, \\ Pennsylvania 15213 \\ Pak Kin Wong \\ SAE Technology Center, Hong Kong Science Park, Shatin, New Territories, Hong Kong \\ Andrew J. Gellman ${ }^{\text {a) }}$ \\ Department of Chemical Engineering, Carnegie Mellon University, 5000 Forbes Ave., Pittsburgh, \\ Pennsylvania 15213 and Institute for Advanced Energy Studies, National Energy Technology Laboratory, \\ 620 Cochrans Mill Rd., Pittsburgh, Pennsylvania 15236
}

(Received 11 September 2009; accepted 26 July 2010; published 3 September 2010)

\begin{abstract}
Thin diamond-like carbon films were subjected to fluorination with gaseous $\mathrm{XeF}_{2}$ under ultrahigh vacuum conditions in order to increase the work function of the diamond-like carbon surface. Changes in the work function and surface composition were monitored with UV photoemission spectroscopy and $\mathrm{x}$-ray photoemission spectroscopy, respectively. Successive $\mathrm{XeF}_{2}$ exposures raised the work function by as much as $1.55 \mathrm{eV}$. Surprisingly, approximately half of the increase in the work function occurred while the coverage of fluorine remained below 0.02 monolayers (ML). This suggests that initial doses of $\mathrm{XeF}_{2}$ remove extrinsic adsorbates from the diamond-like carbon film and that fluorine desorbs with the reaction products. Increasing the exposure of the diamond-like carbon to $\mathrm{XeF}_{2}$ leads to the expected covalent fluorination of the surface, which saturates at fluorine coverages of $6 \mathrm{~F}$ atoms $/ \mathrm{nm}^{2}(\sim 0.3 \mathrm{ML})$. Annealing of the diamond-like carbon to temperatures above $850 \mathrm{~K}$ was required to reduce the surface fluorine concentration to undetectable levels. This did not, however, cause the work function to return to its original, prefluorination value. (C) 2010 American Vacuum Society. [DOI: 10.1116/1.3480335]
\end{abstract}

\section{INTRODUCTION}

Diamond-like carbon (DLC) surfaces are characterized by exceptional hardness, chemical inertness, and low friction coefficient. $^{1-3}$ This has prompted significant interest in using DLC films as protective coatings on a variety of surfaces. One such application of DLC films is as coatings on the sliding surfaces of the read-write heads in hard drives, where the film protects the giant magnetoresistive (GMR) junction from corrosion and mechanical damage from occasional contacts with the disk surface.

In many device applications in which surfaces come into sliding contact with one another, charge transfer is known to occur. ${ }^{4,5}$ This can be detrimental to the performance of the device. The extent of charging is expected to be strongly influenced by the work function of the surface; materials with higher work function provide a higher potential barrier to electrons exiting the material. As a consequence, the modification of the surface of the material to increase its work function will reduce the effects of electron transfer.

One method for increasing the work function of a surface is to modify it by adsorption of an electronegative species. The adsorption of an electronegative species creates an electrical double layer with the outer surface charged negatively. This produces a dipole field at the surface that opposes elec-

a) Author to whom correspondence should be addressed; electronic mail: gellman@cmu.edu tron transport across the surface from the bulk into vacuum, ${ }^{6-8}$ effectively increasing the work function. Fluorine is the most electronegative element and, hence, it is expected that the adsorption of fluorine onto DLC surfaces will increase the surface work function. The chemisorption of fluorine on polycrystalline and single-crystal diamond has been previously studied by several groups. ${ }^{9-12}$ Molecular fluorine and $\mathrm{XeF}_{2}$ were used as fluorinating agents. Generally, covalent functionalization levels of up to 2 monolayers (ML) are reported, with the adsorbed species desorbing over a broad temperature range, 300-1300 K, during heating.

The aim of the study presented here was to increase the work function of DLC films via fluorination of their surfaces under UHV conditions. The fluorinated samples were characterized with X-ray photoemission spectroscopy (XPS) and UV photoemission spectroscopy (UPS). This work shows that fluorination of the DLC surface will lead to significant increases in the work function of the surface. It also provides some insight into the surface chemical processes occurring during fluorination.

\section{EXPERIMENT}

The experiments were performed in a stainless steel UHV chamber with a base pressure of $1.5 \times 10^{-10}$ Torr pumped by a $550 \mathrm{l} / \mathrm{s}$ turbomolecular pump. The chamber is equipped with a $120 \mathrm{~mm}$ diameter hemispherical electron energy analyzer (Omicron EA-125) with a multichannel detector. 
UPS spectra were acquired using the He I line $(21.2 \mathrm{eV})$ from a differentially pumped $\mathrm{He}$ arc discharge source (Specs UVS 10/35). The surface work function was determined from the secondary electron cutoff in the UPS spectra. During the acquisition of UPS spectra the sample was biased to $-10 \mathrm{~V}$ relative to the analyzer at ground potential. The work function of the analyzer, necessary for calculating the sample work function from UPS spectra, was determined from a calibration using a clean $\mathrm{Pt}(111)$ surface of known work function.

The samples coated with DLC films were produced by SAE Magnetics. Their layer-by-layer composition simulated the layered structure of GMR read-write heads. A $\mathrm{TiC}-\mathrm{Al}_{2} \mathrm{O}_{3}$ conductive ceramic was used as a substrate onto which successive layers of $\mathrm{Ti}, \mathrm{Si}$, and then the DLC were deposited. The DLC layer was formed using an arc discharge graphite source coupled with a quadrupole filter. The filter only allowed singly charged carbon atoms to pass and be deposited onto the substrate, while particles consisting of two or more carbon atoms were rejected. The thickness of the DLC overcoat layer was $\sim 20 \AA$. The DLC deposition process is one that has been developed for the production of commercial read-write heads. The films generated by this process have been rigorously tested and qualified for commercial use, ensuring that they are continuous and free of pinholes that might serve as nucleation points for corrosion of the head materials. The DLC overcoats used in this work were supplied from a commercial manufacturer and not fully characterized; however, past experience in studies of fluoroether lubricant adsorption on similar films provided by various commercial manufacturers has shown that there is a great deal of consistency among them, independent of their source of origin. ${ }^{13-23}$

A $1 \mathrm{~cm}^{2}$ square sample with the DLC film deposited on one side was mounted inside the UHV chamber on a Ta support plate. The plate could be heated resistively and cooled with liquid nitrogen through thermal contact with a liquid nitrogen cold finger. The sample temperature could be controlled in the 100-950 K range. Before the experiments, the system with the sample was baked out at 350-370 K for $36 \mathrm{~h}$ under UHV conditions.

The sample surfaces were not cleaned by either ion sputtering or annealing. Such cleaning processes would result in irreversible changes to the $20 \AA$ thick DLC film, and the surface would no longer yield data relevant to the DLC films used on GMR read-write heads.

The Al $K_{\alpha}$ line $(1486.6 \mathrm{eV})$ from a Specs XR-50 x-ray source was used to record $\mathrm{x}$-ray photoemission spectra. XPS analysis of the sample prior to experiments indicates the expected presence of $\mathrm{C}$ and signals from $\mathrm{Ti}, \mathrm{Si}, \mathrm{Al}$, and $\mathrm{O}$ arising from the substrate onto which the diamond-like carbon has been deposited. No significant changes in the XPS signatures for these elements were seen after fluorinating the sample, indicating that they are not reacting with the $\mathrm{XeF}_{2}$. This is indicative of a well formed diamond-like carbon film with no pinholes.

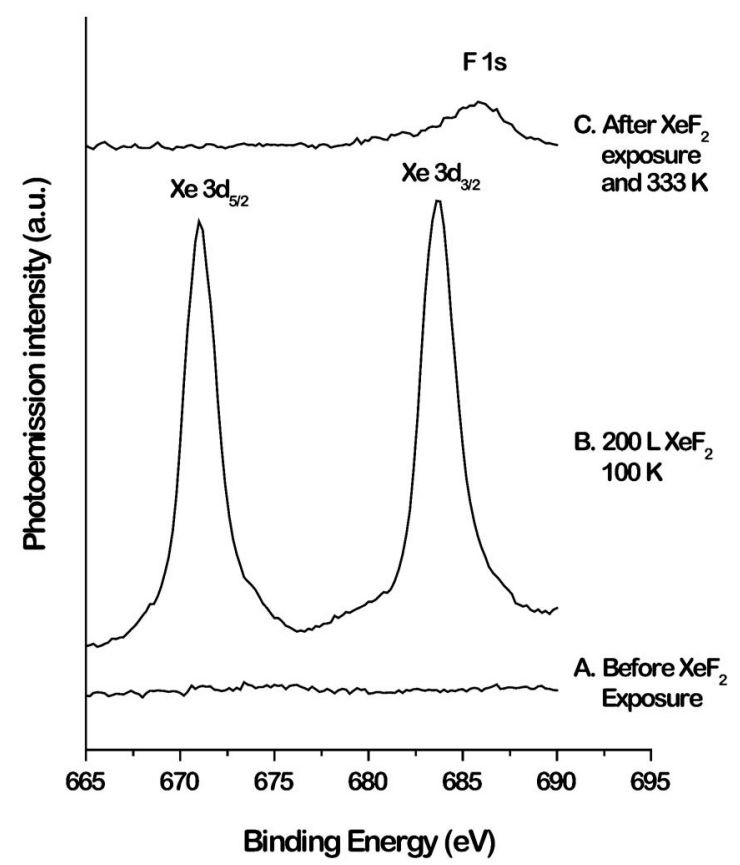

FIG. 1. XPS spectra of the DLC surface (a) at $100 \mathrm{~K}$ and before exposure to $\mathrm{XeF}_{2}$, (b) at $100 \mathrm{~K}$ after exposure to $200 \mathrm{~L}$ of $\mathrm{XeF}_{2}$, and (c) after annealing to $333 \mathrm{~K}$ to desorb excess $\mathrm{XeF}_{2}$. After annealing some fluorine is left on the DLC surface.

Crystalline $\mathrm{XeF}_{2}$ (99.99\% purity) was acquired from Sigma Aldrich and placed into an evacuated glass container connected to the UHV system through a leak valve. Before introducing the $\mathrm{XeF}_{2}$ vapor into the UHV chamber, purification of $\mathrm{XeF}_{2}$ was performed by evacuating the gaseous phase above the $\mathrm{XeF}_{2}$ crystals, removing any contaminant gases that might accumulate in the vial. $\mathrm{XeF}_{2}$ has a vapor pressure of 4.5 Torr at room temperature. ${ }^{24}$ The exposure of the sample to $\mathrm{XeF}_{2}$ was conducted by dynamically backfilling the UHV chamber with $\mathrm{XeF}_{2}$ vapor to pressures of $10^{-7}-10^{-5}$ Torr for a predetermined amount of time.

\section{RESULTS AND DISCUSSION}

Fluorination of the DLC surface was performed on two identical samples using two methods. In one method of fluorination, the sample was held at $100 \mathrm{~K}$ during the exposure to $\mathrm{XeF}_{2}$ vapor. As a result, $\mathrm{XeF}_{2}$ condensed onto the DLC surface. The sample was then heated to $333 \mathrm{~K}$, causing unreacted $\mathrm{XeF}_{2}$ to desorb. In the other fluorination method, the sample was held at $300 \mathrm{~K}$ while being exposed to $\mathrm{XeF}_{2}$ vapor. The sample was then heated to $333 \mathrm{~K}$ before the UPS and XPS spectra were obtained. As expected, the low temperature condensation method produced more efficient fluorination per unit of exposure, as it effectively confined $\mathrm{XeF}_{2}$ at the surface. However, in all other respects, the results of fluorination were similar.

XPS was used to monitor the deposition of $\mathrm{F}$ onto the DLC surface during exposure to $\mathrm{XeF}_{2}$. Figure 1 shows representative XPS spectra of the DLC surface in the range encompassing the $\mathrm{Xe} 3 d$ and $\mathrm{F} 1 s$ lines at three stages in the low temperature exposure sequence. The lower two were 


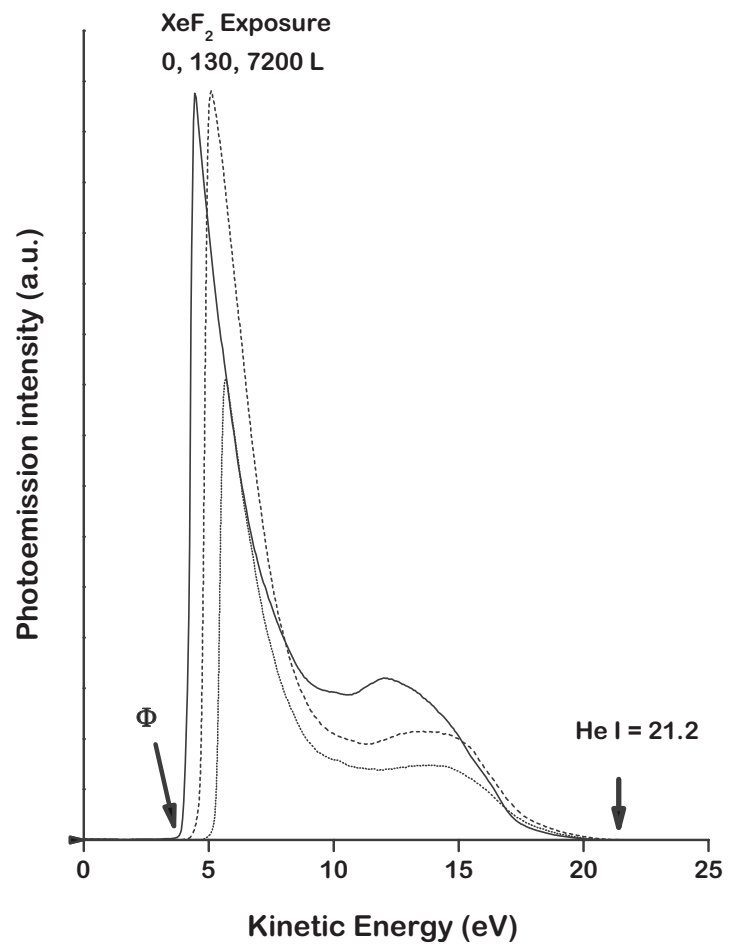

FIG. 2. UPS spectra of the DLC surface before and following increasing exposure to $\mathrm{XeF}_{2}$ at $300 \mathrm{~K}$. The data are plotted such that the electrons emitted from the Fermi level have the energy of the He I photon $(21.2 \mathrm{eV})$. The onset of the secondary electron peak then corresponds to the surface work function. The increase in the work function of the DLC surface upon fluorination with $\mathrm{XeF}_{2}$ is indicated by the shift in the secondary electron onset energy.

obtained with the DLC held at $100 \mathrm{~K}$. After the sample was exposed to $\mathrm{XeF}_{2}$, the doublet of $\mathrm{Xe} 3 d$ lines developed, with F $1 s$ line seen as a shoulder at $687 \mathrm{eV}$ on the higher binding energy $\mathrm{Xe} 3 d_{3 / 2}$ peak. After the sample temperature was raised to $333 \mathrm{~K}, \mathrm{XeF}_{2}$ was completely removed, leaving only the fluorine chemically bound to the surface, revealing the F $1 s$ feature at $686 \mathrm{eV}$.

Fluorine functionalization of the DLC surface leads to the creation of a surface dipole layer. A simple electrostatic model predicts that when the outermost layer of the surface is negatively charged, as it is when modified by adsorbed $\mathrm{F}$ atoms, the work function should increase by

$$
\Delta \Phi=\frac{\sigma \cdot r}{\varepsilon_{0}},
$$

where $\sigma$ is the surface charge density and $r$ is the separation between the charged layers (see, for instance, the discussion in Ref. 8). If the outer layer is positively charged, then the work function will decrease by the same magnitude.

The predicted change in the work function resulting from fluorination of the DLC surface was detected by UPS measurements. Shown in Fig. 2 is a set of typical UPS spectra demonstrating the increase in the surface work function upon fluorination by exposure to $\mathrm{XeF}_{2}$ at $300 \mathrm{~K}$. When the data are plotted so that the energy of electrons emitted from the Fermi level is set equal to the photon energy $(21.2 \mathrm{eV})$, the lowenergy onset of secondary electron emission indicates the magnitude of surface work function. After the two exposures to $\mathrm{XeF}_{2}$ shown in Fig. 2, the work function increased by 0.85 and $1.5 \mathrm{eV}$ relative to the untreated surface.

In order to determine the coverage of fluorine from the intensity of the F $1 s$ peak in the XPS spectra, an instrumentspecific sensitivity factor must be found from an independent calibration. We determined the sensitivity factor by measuring the area of the XPS C $1 s$ peak from the $20 \AA$ thick DLC film on the sample. By knowing the extinction length of the emitted electrons in the DLC film, $\lambda_{C}[2.8 \mathrm{~nm}$ (Ref. 25)], and the ratio of XPS sensitivities for $\mathrm{F}$ and $\mathrm{C}$ atoms, $R$ [1:0.296 (Ref. 26)], the coverage of $\mathrm{F}$ can be calculated from the areas of the $\mathrm{C} 1 s$ and $\mathrm{F} 1 s$ peaks after background subtraction as follows:

$$
\theta_{\mathrm{F}}=I_{\mathrm{F}} \cdot J=\frac{I_{\mathrm{F}}}{I_{\mathrm{C}}} \cdot \frac{D_{\mathrm{C}} \cdot \lambda_{\mathrm{C}} \cos \alpha}{R}\left[1-\exp \left(\frac{-L_{\mathrm{C}}}{\lambda_{\mathrm{C}} \cos \alpha}\right)\right] .
$$

Here, $J$ is the sought conversion factor, $\theta_{\mathrm{F}}$ is the areal density of $\mathrm{F}$ atoms, $D_{\mathrm{C}}$ is the volumetric density of $\mathrm{C}$ atoms in the DLC film (175.6 C/nm $\mathrm{nm}^{3}$, assuming diamond structure), $I_{\mathrm{F}}$ is the integrated signal of the F $1 s$ peak in the XPS spectrum, $I_{\mathrm{C}}$ is the integrated signal of the C $1 s$ peak in the XPS spectrum, $\alpha$ is the angle between surface normal and direction toward the XPS analyzer, and $L_{\mathrm{C}}$ is the thickness of the carbon film $(2.0 \mathrm{~nm})$. If the configuration of the XPS system does not change from experiment to experiment, $J$ needs to be calculated only once.

The fluorine coverage on the DLC surface grows with increasing $\mathrm{XeF}_{2}$ exposure [Fig. 3(B)] until it saturates at $\sim 6 \mathrm{~F} / \mathrm{nm}^{2}$. For comparison, the density of carbon atoms on the diamond (111) surface is $18.6 \mathrm{C} / \mathrm{nm}^{2}$ (which we define as $1 \mathrm{ML}$ ). With each carbon atom having a single unbonded orbital protruding from the (111) surface, this number also corresponds to the maximum theoretical fluorine coverage. In practice, however, fluorination of the clean diamond (111) surface only produces coverages of $\sim 14$ atoms $/ \mathrm{nm}^{2}$ $(\sim 0.75 \mathrm{ML}) .{ }^{10}$ The nominal maximum coverage achieved in our experiments is $\sim 6 \mathrm{~F} / \mathrm{nm}^{2}$ which corresponds to roughly $0.3 \mathrm{ML}$. The fact that this is lower than the coverage observed on diamond can be due to the more disordered nature of the DLC surface.

The work function of the DLC surface, as measured by UPS, increases monotonically as the cumulative exposure to $\mathrm{XeF}_{2}$ increases [Fig. 3(A)], and at an exposure of $7000 \mathrm{~L}$, it reaches $5.29 \mathrm{eV}$, which is $1.55 \mathrm{eV}$ above the initial value. Remarkably, roughly half of the increase $(0.8 \mathrm{eV})$ occurs while the coverage of fluorine remains at levels not detectable by the XPS. We estimate the sensitivity limit of the XPS detection at $5 \%$ of the maximum fluorine coverage or $\sim 0.3 \mathrm{~F} / \mathrm{nm}^{2}(\sim 0.015 \mathrm{ML})$.

We propose that the increase in the work function that is not accompanied by a measurable increase in fluorine coverage is caused by the initial doses of $\mathrm{XeF}_{2}$ oxidizing the DLC surface or species adsorbed on it to yield fluorine-containing reaction products that are volatile and desorb, leaving no covalently bound fluorine. An alternative interpretation of the initial sensitivity of the work function to $\mathrm{XeF}_{2}$ exposure 


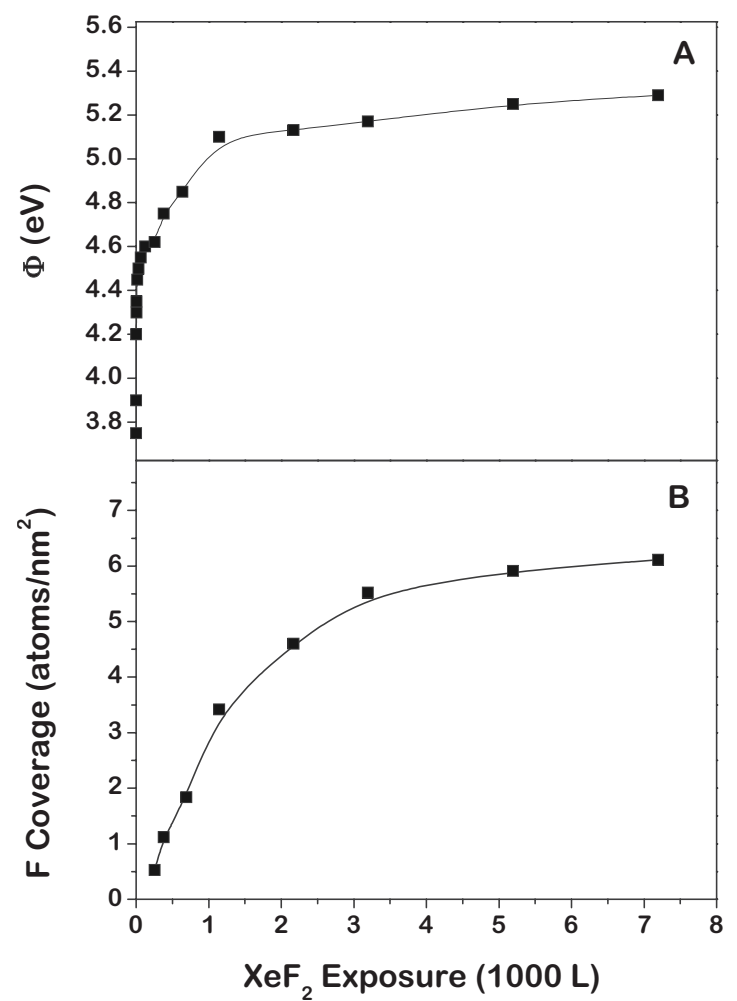

FIG. 3. (A) Change in DLC surface work function and (B) the coverage of chemisorbed fluorine as a function of exposure to $\mathrm{XeF}_{2}$ at $300 \mathrm{~K}$. Initially, multiple small exposures result in no detectable increase in fluorine concentration measured by XPS (points not shown on B), but the work function increases rapidly. Solid lines are drawn to guide the eye.

could be that there is a small number of highly reactive carbon sites that has a disproportionately large effect on the surface work function. Strongly bound, low-concentration fluorine species were observed by Foord et al. ${ }^{9}$ on polycrystalline diamond surfaces deposited by chemical vapor deposition. However, they observed the F $1 s$ peak of this fluorine species at $688 \mathrm{eV}$, which was a significantly higher binding energy than the primary F $1 s$ peak at $685.5 \mathrm{eV}$. In the work described here, only the F $1 s$ peak at $685.5 \mathrm{eV}$ was observed. In addition, an estimate using Eq. (1) shows that at a fluorine coverage equal to the detection limit of our XPS system, the fluorine on the surface cannot produce a $0.8 \mathrm{eV}$ shift in the work function. Assuming an excess of $0.4 e^{-}$on the $\mathrm{F}$ and an identical charge deficit on the $\mathrm{C}$ atoms, ${ }^{27}$ a C-F bond length of $0.134 \mathrm{~nm}$, and a bond orientation perpendicular to the surface, one arrives at the maximum possible work function increase of $0.3 \mathrm{eV}$ at a fluorine coverage of $\sim 0.3 \mathrm{~F} / \mathrm{nm}^{2}$. Therefore, we conclude that this low exposure increase of the work function is not due to adsorbed fluorine atoms.

Annealing of the fluorinated DLC surface causes desorption of fluorine (or fluorine-containing functionalities) at temperatures as low as $373 \mathrm{~K}$. The sample was annealed at increasing temperatures separated by $50 \mathrm{~K}$ increments, and the loss of surface fluorine was followed by observing the decrease in the intensity of the F $1 s$ XPS signal (Fig. 4). The sample was held for $10 \mathrm{~min}$ at each temperature and then

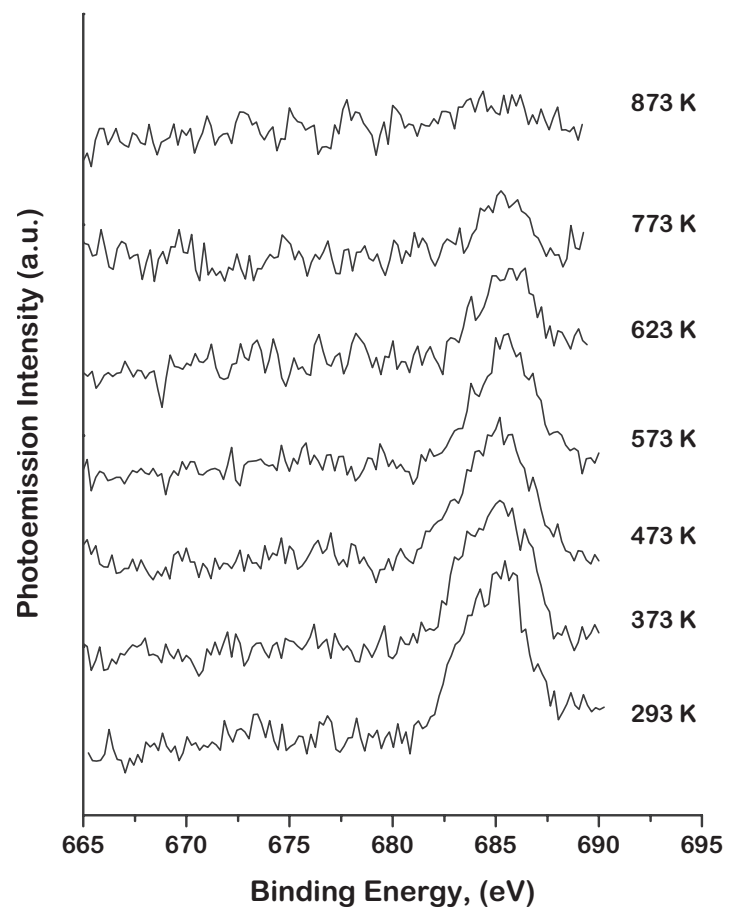

FIG. 4. XPS spectra of the fluorinated DLC film following annealing to increasing temperatures. The F $1 s$ signal decreases as the anneal temperature is increased. For clarity, XPS spectra are shown at only half of the annealing temperatures used.

cooled to $300 \mathrm{~K}$ prior to making the XPS measurements. Almost all of the fluorine is desorbed after the sample has been annealed at $873 \mathrm{~K}$. No significant shift in the $\mathrm{F} 1 s$ peak position is observed as a result of annealing.

Figure 5 summarizes the work function changes as a function of fluorine coverage on the DLC surface during $\mathrm{XeF}_{2}$ exposures and subsequent annealing. As the $\mathrm{F}$ coverage in-

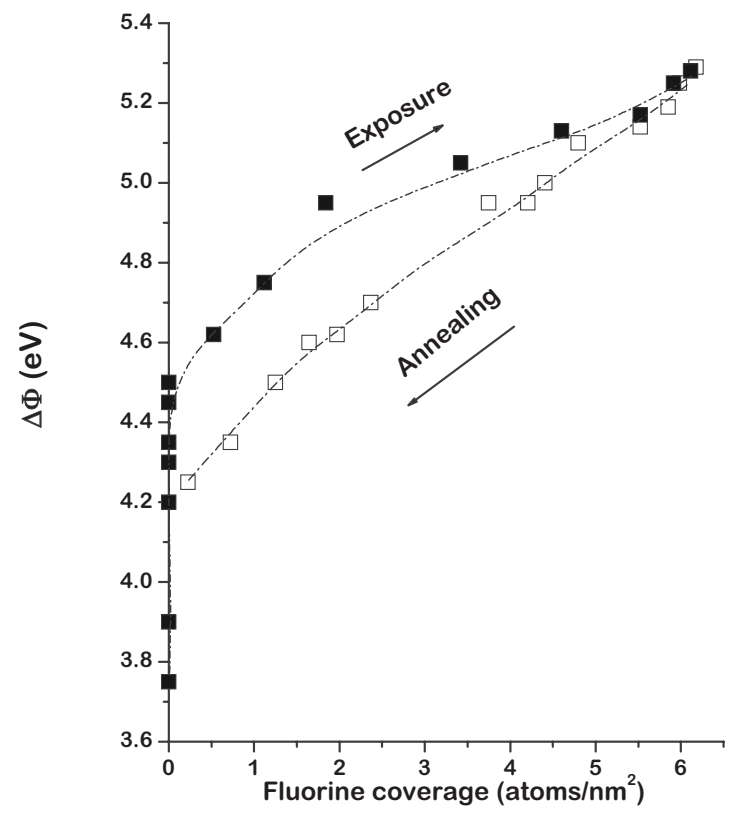

FIG. 5. Work function of the DLC film vs $\mathrm{F}$ coverage during $\mathrm{XeF}_{2}$ exposure at $300 \mathrm{~K}$ and following annealing at temperatures up to $873 \mathrm{~K}$. 
creases during $\mathrm{XeF}_{2}$ exposure, the work function increases. As the $\mathrm{F}$ coverage decreases during annealing, the work function decreases. Mismatch between the exposure and annealing curves indicates that annealing induces changes other than just reversible F depletion from the surface. At the lowest coverage reached by annealing to $873 \mathrm{~K}$, the work function stays above the prefluorination level. The work function drops to $4.2 \mathrm{eV}$, which is below the $4.5 \mathrm{eV}$ value achieved after the initial exposures to $\mathrm{XeF}_{2}$ that left no detectable fluorine on the surface. This behavior could result from structural changes occurring in the DLC film at elevated temperatures. Unfortunately, annealing of DLC films to high temperatures produces irreversible changes in their structure and composition and thus, further exposure of the annealed DLC film to $\mathrm{XeF}_{2}$ would not provide any insights relevant to the surface chemistry of the as deposited DLC films.

\section{CONCLUSIONS}

Fluorination of DLC films with $\mathrm{XeF}_{2}$ results in an increase of $1.55 \mathrm{eV}$ in the surface work function. This is sufficient to cause significant reduction in electron transfer from the surface. Initial exposures to $\mathrm{XeF}_{2}$ result in a $0.8 \mathrm{eV}$ increase in the work function without any detectable deposition of fluorine. This is probably the result of $\mathrm{XeF}_{2}$ initially fluorinating and removing extraneous adsorbed species from the carbon surface. Further increase in the surface work function is accompanied by rising fluorine coverage. The removal of adsorbed fluorine by annealing at $870 \mathrm{~K}$ lowers the work function.

\section{ACKNOWLEDGMENTS}

This work was supported by the Data Storage Systems Center at Carnegie Mellon with funding from SAE Magnetics.
${ }^{1}$ A. Erdemir and C. Donnet, J. Phys. D 39, R311 (2006).

${ }^{2}$ J. Robertson, Mater. Sci. Eng. R. 37, 129 (2002).

${ }^{3}$ A. Grill, Diamond Relat. Mater. 8, 428 (1999).

${ }^{4}$ T. J. Harvey, R. J. K. Wood, G. Denuault, and H. E. G. Powrie, Tribol. Int. 35, 605 (2002).

${ }^{5}$ J. D. Kiely and Y. T. Hsia, J. Appl. Phys. 91, 4631 (2002).

${ }^{6}$ H. Ago, T. Kugler, F. Cacialli, W. R. Salaneck, M. S. P. Shaffer, A. H. Windle, and R. H. Friend, J. Phys. Chem. B 103, 8116 (1999).

${ }^{7}$ I. D. Baikie, U. Petermann, A. Speakman, B. Lagel, K. M. Dirscherl, and P. J. Estrup, J. Appl. Phys. 88, 4371 (2000).

${ }^{8}$ T. C. Leung, C. L. Kao, W. S. Su, Y. J. Feng, and C. T. Chan, Phys. Rev. B 68, 195408 (2003).

${ }^{9}$ J. S. Foord, N. K. Singh, R. B. Jackman, A. Gutierrez-Sosa, S. Proffitt, and K. B. Holt, Surf. Sci. 488, 335 (2001).

${ }^{10}$ A. Freedman, J. Appl. Phys. 75, 3112 (1994).

${ }^{11}$ Y. Mitsuda, T. Yamada, T. J. Chuang, H. Seki, R. P. Chin, J. Y. Huang, and Y. R. Shen, Surf. Sci. 257, L633 (1991).

${ }^{12}$ T. Yamada, H. Seki, and T. J. Chuang, Jpn. J. Appl. Phys., Part 1 39, 1826 (2000).

${ }^{13}$ L. Cornaglia and A. J. Gellman, J. Vac. Sci. Technol. A 15, 2755 (1997).

${ }^{14}$ L. Cornaglia and A. J. Gellman, Adv. Inf. Storage Syst. 8, 57 (1998).

${ }^{15}$ L. Cornaglia, A. J. Gellman, S. Howe, and S. Nadimpalli, ASME Symposium on Tribology of Contact/Near Contact Recording for Ultra-high Density Magnetic Storage, San Francisco, CA, edited by S. Bhatia and A. K. Menon (ASME, New York, 1996), pp. 38-44.

${ }^{16}$ A. J. Gellman, Curr. Opin. Colloid Interface Sci. 3, 368 (1998).

${ }^{17}$ A. J. Gellman, K. Paserba, and N. Vaidyanathan, Tribol. Lett. 12, 111 (2002).

${ }^{18}$ R. Z. Lei and A. J. Gellman, Langmuir 16, 6628 (2000).

${ }^{19}$ R. Z. Lei, K. Paserba, A. J. Gellman, N. Shukla, and L. Cornaglia, ASME Symposium on Nanotribology and Nanotechnology for $1 \mathrm{Tbit} / \mathrm{in}^{2}$, San Francisco, CA, 2001 (unpublished).

${ }^{20}$ K. Paserba, N. Shukla, A. J. Gellman, J. Gui, and B. Marchon, Langmuir 15, 1709 (1999).

${ }^{21}$ N. Shukla and A. J. Gellman, J. Vac. Sci. Technol. A 18, 2319 (2000).

${ }^{22}$ N. Shukla, A. J. Gellman, and J. Gui, Langmuir 16, 6562 (2000).

${ }^{23}$ N. Shukla, J. Gui, and A. J. Gellman, Langmuir 17, 2395 (2001).

${ }^{24}$ M. Tramšek and B. Žemva, Acta Chim. Slov. 53, 105 (2006).

${ }^{25}$ P. J. Cumpson and M. P. Seah, Surf. Interface Anal. 25, 430 (1997).

${ }^{26}$ J. Moulder, W. Stickle, P. Sobol, and K. Bomben, Handbook of X-Ray Photoelectron Spectroscopy (Perkin-Elmer Corporation, Eden Prairie, 1992).

${ }^{27}$ M. M. C. Ferreira and E. Suto, J. Phys. Chem. 96, 8844 (1992). 